\title{
Diagnosis of double aortic arch by cross sectional echocardiography with Doppler colour flow mapping
}

\author{
MEE-NIN KAN, NAVIN C NANDA, A R STOPA \\ From the Division of Cardiovascular Disease, University of Alabama at Birmingham, USA
}

SUMMARY A case of double aortic arch in a 15 month old boy was diagnosed by cross sectional echocardiography supplemented by Doppler colour flow mapping. These examinations showed two very large vessels communicating with the aorta; the demonstration of anterograde flow in one vessel and retrograde flow in the other enabled a definite diagnosis of Edwards type IA double aortic arch to be made.

Early surgical intervention is often required in infants and children with double aortic arch who show symptoms caused by tracheal or oesophageal compression. ${ }^{1}$ Definite diagnosis is made by aortography, which is an invasive procedure. Real time cross sectional echocardiography is a useful noninvasive method of detecting this anomaly, ${ }^{23}$ but the diagnosis depends on several factors such as ease of obtaining a suprasternal examination and identification of the oesophagus, both of which may be difficult in a small child. Also it may be difficult to discriminate between a bifurcated aortic arch and a dilated cephalic branch or aberrant vessel arising from a non-bifurcated aortic arch. The diagnosis often relies on exact positioning or orientation of the transducer. Both are subjective and liable to error. We present a case in which a double aortic arch was diagnosed by cross sectional echocardiography supplemented by Doppler colour flow mapping. We used a high parasternal approach rather than the previously used subcostal or suprasternal approach.

\section{Case report}

A 15 month old boy presented with moderate respiratory distress and a history of recurrent upper respiratory tract infections. Coarse rhonchi were heard throughout the lung fields. A cross sectional echocardiogram performed through a high parasternal window showed a large vessel, about the same size as

Requests for reprints to Dr Navin C Nanda, Heart Station, SWB/W001, University of Alabama at Birmingham, Birmingham, Alabama 35294, USA. the aortic arch, arising from the ascending aorta. A second vessel, about the same size as the first vessel, was seen joining the upper portion of the descending aorta in the same plane. Colour flow mapping (Aloka 880 system) showed blood flowing from the ascending aorta into the first vessel and moving in an opposite direction from the second vessel into the descending aorta (fig 1).

\section{Discussion}

The cross sectional echocardiographic findings were of two very large vessels communicating with the aorta. Colour Doppler showed blood flowing anterogradely in the first vessel and retrogradely in the second vessel and enabled us to make a definite diagnosis of Edwards type IA double aortic arch. ${ }^{4} \mathrm{Col}-$ our Doppler flow mapping supplemented cross sectional echocardiography and assisted diagnosis. Angiography confirmed the cross sectional echocardiographic findings (fig 2). Surgical resection was successful and the child showed considerable clinical improvement.

\section{References}

1 Gross RE. Arterial malformations which cause compression of the trachea or esophagus. Circulation 1955;11:124-34.

2 Sahn DJ, Valdes-Cruz IM, Quit TW, et al. Twodimensional echocardiography and intravenous digital video subtraction angiography for diagnosis and 

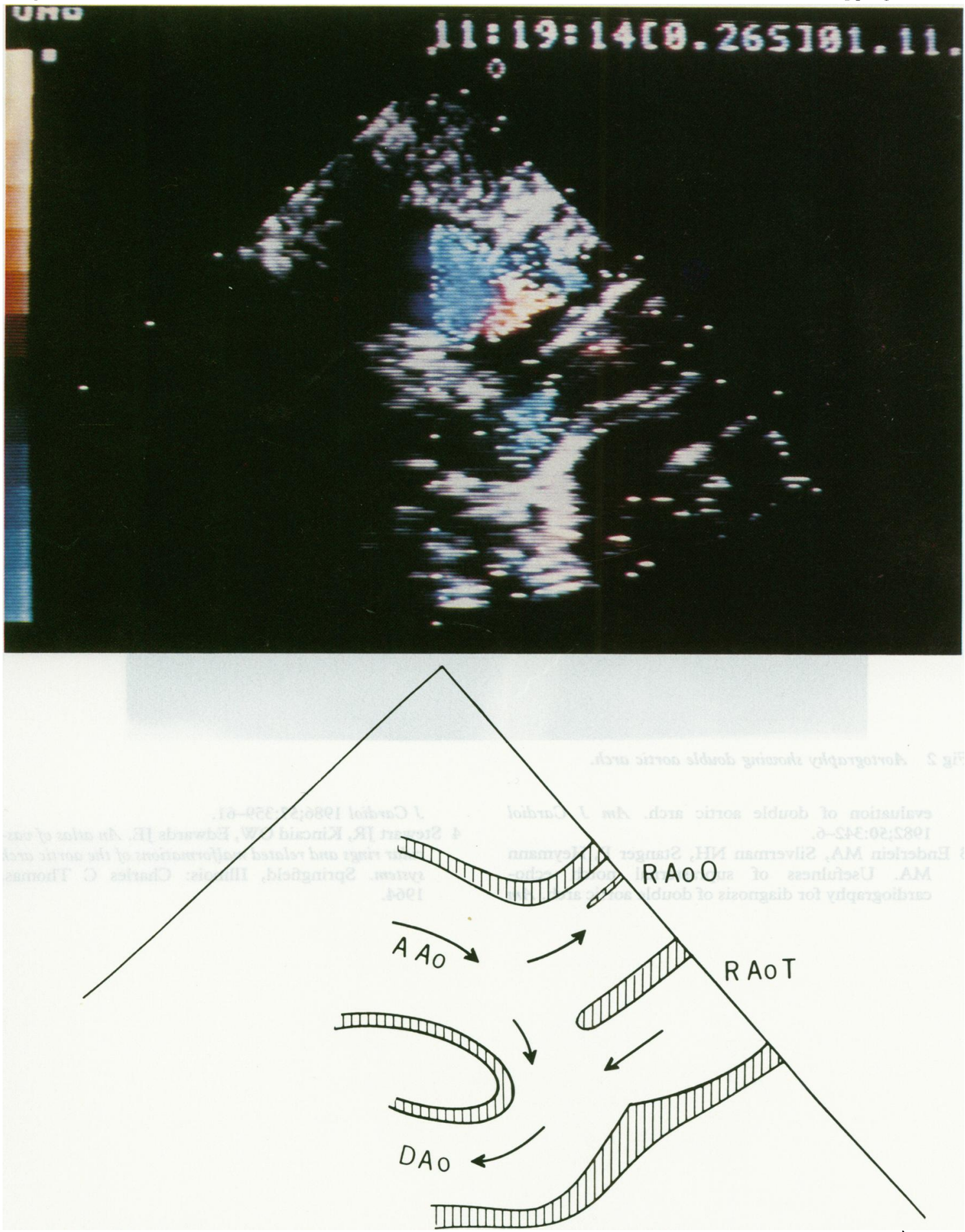

Fig 1 Cross sectional and Doppler colour flow mapping from the high parasternal approach in a case of double aortic arch. Two very large vessels communicated with the aorta. Colour flow study showed blood flowing into the first vessel $(R A o O)$ from the ascending aorta $(A A o)$ and retrogradely from the second vessel (RAoT) into the descending aorta $(D A o)$. Red indicates flow toward the transducer and blue shows flow away from transducer. $R A o O$, origin of the right aortic arch; RAoT, termination of the right aortic arch. 


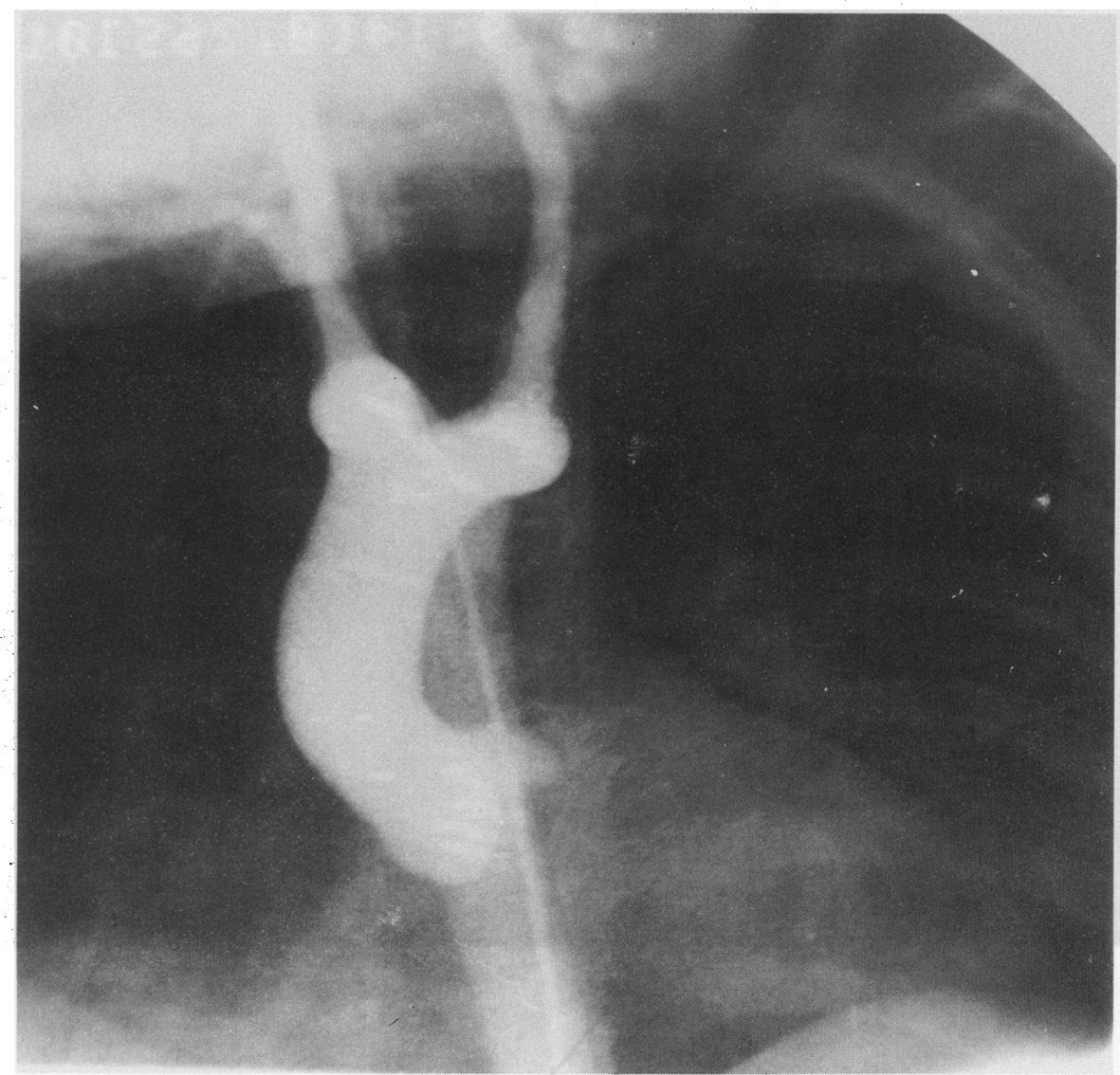

Fig 2 Aortography showing double aortic arch.

evaluation of double aortic arch. Am J Cardiol 1982;50:342-6.

3 Enderlein MA, Silverman NH, Stanger P, Heymann

MA. Usefulness of suprasternal notch echocardiography for diagnosis of double aortic arch. Am
J Cardiol 1986;57:359-61.

4 Stewart JR, Kincaid OW, Edwards JE. An atlas of vascular rings and related malformations of the aortic arch $\overline{0}$ system. Springfield, Illinois: Charles C Thomas, 1964. 